\title{
Cardiovascular Diseases in Acromegaly: Not Diagnosed What is Not Suspected
}

\author{
Ivan Lozada Martinez ${ }^{1 *}$, Daniela Torres Llinás ${ }^{2}$ and Andrés Llamas Nieves ${ }^{2}$ \\ ${ }^{1}$ Director of Medical-Surgical Research Center, School of Medicine, University of Cartagena, Colombia \\ ${ }^{2}$ Researcher, Medical-Surgical Research Center, School of Medicine, University of Cartagena, Colombia \\ *Corresponding author: Ivan David Lozada Martinez, Director of Medical-Surgical Research Center, \\ School of Medicine, University of Cartagena, Cartagena, Colombia
}

Acromegaly has been described as a rare disease, the result of chronic excessive secretion of growth hormone (GH), with subsequent elevation of Insulin-like Growth Factor I (IGF-1) levels [1]. This condition is associated with an increase in morbidity and mortality compared to the general population, without taking into account that this endocrine disorder per se, increases cardiovascular risk by causing metabolic disorders such as hyperglycemia, dyslipidemia and systemic hypertension [2].

Beyond focusing on the endocrine disorder that impacts with physical manifestations, it should be borne in mind that cardiovascular entities may be present asymptomatically. The increase in the GH/IGF-1 index generates persistent sodium retention, with a consequent increase in circulating volume, contributing to the early development of hypertension, hypertrophic heart disease, valvular disease, systolic and diastolic dysfunction [3]. For this reason, it has been found that hypertension affects more than $50 \%$ of patients, being a risk factor for independent mortality [4]. In the series of cases reported, valvular diseases have been found in more than $75 \%$ of the cases, and arrhythmias in more than $90 \%$ of the cases [5], so although the cut-off score of patients with acromegaly and hypertension is reported to be around $50 \%$, it is evidently higher.

No predilection has been found regarding the degree of involvement of cardiovascular disease in acromegaly, with the gender or family history of hypertension, but it is directly proportional to the levels of IGF-1 and GH [6]. Obstructive sleep apnea is another condition found in this disease, and it contributes substantially with the increase of blood pressure, and therefore, the risk of white organ injury due to damage at the level of organic microcirculation. Studies show that the prevalence of left ventricular hypertrophy is up to $78 \%$ in echocardiographic studies, with the heart being the main organ affected, due to the increase of IGF-1 receptors in cardiomyocytes, which stimulates cardiac contractility, with subsequent hypertrophy and regional fibrosis [7].

Stroke is another entity that may go unnoticed, and can manifest itself by progressive damage as the individual advances in age, exhibiting as vascular dementia. Although the incidence and prevalence of brain injury is expected to be higher in this group, it has not been shown to be so [8], however, this does not mean that strict follow-up should be undertaken.

Knowing that arterial wall inflammation is an important mediator of endothelial dysfunction and development of atherosclerotic disease, chronic renal disease and thromboembolic events, they can also be expected in this type of individuals. In this pathophysiological process, immune cells invade the arterial wall and induce expression of adhesion molecules, with subsequent leukocyte adhesion, which creates a pro-inflammatory and pro-atherogenic environment [9]; which together, with hypertensive glomerulopathy, secondary to systemic arterial hypertension, accelerates the glomerular lesion, while at the peripheral level, the destabilization of an atherosclerotic plaque together with inflammatory adhesion cells, precipitates the formation of a thrombus/embolus. 
Based on the above, the constant review of endothelial, arterial and cardiac integrity are the fundamental pillars to maintain cardiovascular risk monitored on its entirety, the use of echocardiography, echocardiogram, carotid doppler, lower limb doppler, and pulse wave velocity measurement (which makes it possible to identify arterial stiffness by measuring the intima/mean thinning and flow-mediated dilation) [10] are useful in the review of the cardiovascular system. It is also necessary to evaluate the lipid profile and glycemia, which are the other components that also contribute to endothelial dysfunction. In this order of ideas, acromegaly is a multifactorial condition that carries an elevated cardiovascular risk, so its detailed evaluation is a fundamental aspect to control the risk of morbidity and mortality.

\section{Financial Support}

None.

\section{Conflict of Interest}

None.

\section{Authors Contribution}

All authors have contributed for this manuscript.

\section{References}

1. Puglisi $S$, Ferraù $F$, Ragonese $M$, Spagnolo $F$, Cannavò $S$ (2020) Cardiometabolic risk in acromegaly: A review with a focus on pasireotide. Front Endocrinol 11: 1-7.

2. Berg C, Petersenn S, Lahner H, Herrmann BL, Buchfelder
M, et al. (2010) Cardiovascular risk factors in patients with uncontrolled and long-term acromegaly: Comparison with matched data from the general population and the effect of disease control. J Clin Endocrinol Metab 95: 3648-3656.

3. Kamenicky P, Blanchard A, Frank M, Salenave S, Letierce A, et al. (2011) Body fluid expansion in acromegaly is related to enhanced epitelial sodium channel $(\mathrm{ENaC})$ activity. $J$ Clin Endocrinol Metab 96: 2127-2135.

4. Mosca S, Paolillo S, Colao A, Bossone E, Cittadini A, et al. (2013) Cardiovascular involvement in patients affected by Acromegaly: An appraisal. Int J Cardiol 167: 1712-1718.

5. Colao A, Pivonello R, Grasso LFS, Auriemma RS, Galdiero $M$, et al. (2011) Determinants of cardiac disease in newly diagnosed patients with acromegaly: Results of a 10 year survey study. Eur J Endocrinol 165: 713-721.

6. Gadelha MR, Kasuki L, Lim DST, Fleseriu M (2019) Systemic complications of acromegaly and the impact of the current treatment landscape: An update. Endocr Rev 40: 268-332.

7. Ramos-Levi AM, Marazuela M (2019) Bringing cardiovascular comorbidities in acromegaly to an update. How should we diagnose and manage them? Front Endocrinol 10: 120.

8. Kasuki L, da Silva P, Baranski E, Roberto M (2019) Determinants of morbidities and mortality in acromegaly. Arch Endocrinol Metab 63: 630-637.

9. Wolters TLC, van der Heijden CDCC, van Leeuwen N, Hijmans-Kersten BTP, Netea MG, et al. (2019) Persistent inflammation and endothelial dysfunction in patients with treated acromegaly. Endocr Connect 8: 1553-1567.

10. Maffei P, Dassie F, Wennberg A, Parolin M, Vettor R (2019) The endothelium in acromegaly. Front Endocrinol 10: 437. 